\title{
Therapeutic efficacy of sonic hedgehog protein in experimental diabetic neuropathy
}

\author{
Nigel A. Calcutt, ${ }^{1}$ Karen L. Allendoerfer ${ }^{2}$ Andrew P. Mizisin, ${ }^{1}$ Alicia Middlemas, ${ }^{3}$ \\ Jason D. Freshwater, ${ }^{1}$ Monica Burgers,${ }^{1}$ Rigel Ranciato, ${ }^{2}$ Jean-Dominique Delcroix, ${ }^{3}$ \\ Frederick R. Taylor, ${ }^{4}$ Renee Shapiro, ${ }^{4}$ Kathy Strauch, ${ }^{4}$ Henryk Dudek, ${ }^{2}$ \\ Thomas M. Engber, ${ }^{4}$ Alphonse Galdes, ${ }^{4}$ Lee L. Rubin, ${ }^{2}$ and David R. Tomlinson ${ }^{3}$ \\ ${ }^{1}$ Department of Pathology, University of California at San Diego, La Jolla, California, USA \\ ${ }^{2}$ Curis Inc., Cambridge, Massachusetts, USA \\ ${ }^{3}$ Division of Neuroscience, School of Biological Sciences, University of Manchester, Manchester, United Kingdom \\ ${ }^{4}$ Biogen Inc., Cambridge, Massachusetts, USA
}

\begin{abstract}
Hedgehog proteins modulate development and patterning of the embryonic nervous system. As expression of desert hedgehog and the hedgehog receptor, patched-1, persist in the postnatal and adult peripheral nerves, the hedgehog pathway may have a role in maturation and maintenance of the peripheral nervous system in normal and disease states. We measured desert hedgehog expression in the peripheral nerve of maturing diabetic rats and found that diabetes caused a significant reduction in desert hedgehog mRNA. Treating diabetic rats with a sonic hedgehog-IgG fusion protein fully restored motor- and sensory-nerve conduction velocities and maintained the axonal caliber of large myelinated fibers. Diabetes-induced deficits in retrograde transport of nerve growth factor and sciatic-nerve levels of calcitonin gene-related product and neuropeptide $Y$ were also ameliorated by treatment with the sonic hedgehog-IgG fusion protein, as was thermal hypoalgesia in the paw. These studies implicate disruption of normal hedgehog function in the etiology of diabetes-induced peripheral-nerve dysfunction and indicate that delivery of exogenous hedgehog proteins may have therapeutic potential for the treatment of diabetic neuropathy.
\end{abstract}

J. Clin. Invest. 111:507-514 (2003). doi:10.1172/JCI200315792.

\section{Introduction}

The mammalian hedgehog $(\mathrm{Hh})$ protein family (sonic, desert, and indian) are relatives of a Drosophila hedgehog protein that regulates segmental polarity during development and are expressed at many sites in the developing mouse embryo (1). Within the nervous system, sonic hedgehog protein $(\mathrm{SHh})$ is associated with development and patterning of the central nervous system (2-5). In contrast, the morphogenic effects of desert hedgehog protein $(\mathrm{DHh})$ are restricted to the peripheral nervous

Received for publication April 25, 2002, and accepted in revised form December 4, 2002

Address correspondence to: Nigel A. Calcutt, Department of Pathology, University of California at San Diego, La Jolla, California 92093-0612, USA. Phone: (858) 534-5331;

Fax: (858) 534-1886; E-mail: ncalcutt@ucsd.edu.

Conflict of interest: Karen L. Allendoerfer, Rigel Ranciato, Frederick R. Taylor, Renee Shapiro, Kathy Strauch, Henryk Dudek, Thomas M. Engber, Alphonse Galdes, and Lee L. Rubin were full-time employees of Curis Inc. or Biogen Inc. and enrolled in stock option programs when this work was performed.

Nonstandard abbreviations used: hedgehog $(\mathrm{Hh})$; sonic hedgehog protein $(\mathrm{SHh})$; desert hedgehog protein (DHh); streptozotocin (STZ); SHh with the N-terminal cysteine replaced by two isoleucine molecules (II-SHh); II-SHh-IgG1 fusion protein (SHh-IgG); calcitonin gene-related product (CGRP); neuropeptide Y (NPY); nerve growth factor (NGF); motor-nerve conduction velocity (MNCV); sensory-nerve conduction velocity (SNCV); ciliary neuronotrophic factor (CNTF); patched (Ptc); smoothened (Smo). system and appear to focus specifically on cellular elements of the epi- and perineurial sheaths (6). Deletion of the $d h b$ gene that codes for DHh in mice results in disruption of the fascicular structure of peripheral nerves, with development of a thin and disorganized perineurial sheath and an increase in blood-nerve barrier permeability that is associated with decreased connexin- 43 expression by perineurial cells (6). A similar modification of nerve structure was associated with a homozygous missense mutation of the $D H H$ gene in a human diagnosed with "minifascicular neuropathy" (7). These findings point to a role for Hh proteins in the development of the peripheral nervous system.

Recent findings in acute peripheral-nerve injury models have revealed a novel function for hedgehog proteins in adult nerve regeneration and repair. The $s h b$ mRNA was strikingly upregulated in the peripheral nerve after crush injury (8), and exogenous SHh protein administration enhanced the speed of nerve recovery (9). The Hh signaling pathway is also upregulated soon after injury in a model of surgically induced hind limb ischemia, and exogenous SHh protein administration augments blood-flow recovery and limb salvage (10). Emerging evidence therefore suggests that signaling pathways initiated by $\mathrm{Hh}$ proteins are involved in the response to a range of peripheral-nerve lesions and participate in the repair and recovery process. 
Diabetes mellitus is a chronic metabolic disease in which nerves progressively deteriorate. Diabetes causes structural changes to the perineurium of peripheral nerves and a decrease in connexin expression (11-13), which could contribute to the pathogenesis of diabetic neuropathy by interfering with maintenance of the blood-nerve barrier (14). Given the growing appreciation of the role of Hh proteins in perineurial development and recovery of nerves from injury, we examined the expression of $h b$ mRNA in the sciatic nerve of maturing diabetic rats, and we assessed the therapeutic potential of a fusion protein incorporating human recombinant SHh and rat IgG (SHh-IgG) in ameliorating a range of diabetes-induced functional and structural disorders of peripheral nerves.

\section{Methods}

Experimental organization. Three distinct studies were performed in diabetic rats, with studies 1 and 2 undertaken concurrently in two separate laboratories and study 3 performed in the same laboratory as study 2 . For measurements replicated in both laboratories, identical experimental procedures were employed, except where noted below. All procedures were performed after approval by the local animal subjects committee or the equivalent body.

Induction, monitoring, and maintenance of diabetes. Diabetes was induced in male Wistar rats (Charles River, Kent, United Kingdom, or Harlan, San Diego, California, USA) with an intraperitoneal injection $(55 \mathrm{mg} / \mathrm{kg})$ of streptozotocin (STZ; Sigma-Aldrich, St. Louis, Missouri, USA) given after an overnight fast. The drug was freshly dissolved in sterile saline immediately before injection. Three days later, tail-vein blood glucose was measured by a strip-operated reflectance photometer, and any STZ-treated rats with a blood glucose level of less than $15 \mathrm{mmol} / \mathrm{l}$ were removed from the study. Rats were group housed with free access to food and water in a 12-hour light/dark cycle. At the end of each study, blood samples were collected, and plasma glucose levels were measured by spectrophotometric assay using a commercial kit (Sigma-Aldrich).

Drug treatments. A modified form of SHh was constructed in which the N-terminal cysteine of SHh was replaced by two isoleucine molecules (II-SHh) to provide increased potency (15). This was then used to construct an II-SHh-IgG1 fusion protein (SHh-IgG), which was expressed in Pichia pastorus and purified using methyl sulfonate sepharose, protein A sepharose, and carboxy methyl POROS chromatography (Boehringer Mannheim, Indianapolis, Indiana, USA). The intact fusion protein was pooled, filtered, and dialyzed into $2 \times$ PBS for storage. Daily aliquots were prepared at the start of each study, coded so that the experimenters were unaware of the treatment group, and stored at $-70^{\circ} \mathrm{C}$. Aliquots were thawed at room temperature immediately before use and diluted with water to achieve a final vehicle concentration of $1 \times$ PBS. Rats were given SHh-IgG thrice weekly (on Monday, Wednesday, and Friday) at doses of $0.3,1.0$, or
$3.0 \mathrm{mg} / \mathrm{kg}$ subcutaneously to the scruff of the neck. All animals not receiving SHh-IgG were injected with PBS vehicle. Treatments were initiated after 5 weeks of diabetes and maintained for a further 5 weeks so that the total duration of diabetes was 10 weeks.

Behavioral tests. Behavioral studies were performed 24 hours after the last injection of SHh-IgG and after a period of acclimation to the testing apparatus. Thermal response latency was measured using a focused heat source directed onto the plantar surface of the foot (16). Flinching of the hind paw after injection of $50 \mu \mathrm{l}$ of $0.5 \%$ formalin into the dorsum of the ipsilateral foot was counted in 1-minute blocks at 5-minute intervals for 60 minutes (17), and the total number of observed flinches was calculated.

Sciatic-nerve laser Doppler flux. Rats were anesthetized by an intraperitoneal injection $(2 \mathrm{ml} / \mathrm{kg})$ of a solution consisting of pentobarbital $(12.5 \mathrm{mg} / \mathrm{ml})$ and diazepam $(1.25$ $\mathrm{mg} / \mathrm{ml}$ ) in $0.9 \%$ saline, and the right femoral artery was cannulated to allow measurement of blood pressure using a MacLab/8s computer system. The left sciatic nerve was exposed in the mid-thigh region, and wholenerve blood flow was assessed using a laser Doppler flowmeter (TSI, St. Paul, Minnesota, USA). This procedure allows rapid measurement of whole-nerve (epineurial and endoneurial) blood flow without protracted surgery (18). Nerve temperature was maintained at $34^{\circ} \mathrm{C} \pm 1^{\circ} \mathrm{C}$ during the measurement period. The probe tip was placed just above the nerve, and five consecutive readings of local arbitrary flow units were made at $1-\mathrm{mm}$ increments along the nerve before conversion to conductance using femoral-artery blood pressure values. The median of the five values was used to represent flow in the nerve.

Neuropeptide assays. Calcitonin gene-related product (CGRP) and neuropeptide Y (NPY) were measured by radioimmunoassay, and nerve growth factor (NGF) was measured in extracts from segments of sciatic nerve by ELISA exactly as described elsewhere $(19,20)$. Retrograde transport of NGF was measured by placement of double prolene ligatures $1 \mathrm{~cm}$ apart on the sciatic nerve. The ligatures were left in place for 18 hours, and the NGF accumulating distal to both ligatures was measured as described above. The rationale for this procedure - in which retrograde transport is computed from the accumulation distal to the lower ligature, using the accumulation distal to the upper ligature to quantify and subtract the nonmobile fraction - is described in detail elsewhere (19).

Nerve morphometry. Segments of mid-thigh sciatic nerve were removed and fixed overnight at $4^{\circ} \mathrm{C}$ in $2.5 \%$ phosphate-buffered glutaraldehyde, postfixed in $1 \%$ aqueous osmium tetroxide, and dehydrated using a series of graded alcohols and propylene oxide. Nerves were embedded in araldite resin, and $1-\mu \mathrm{m}$ sections were cut and stained with $p$-phenylenediamine before examination with a light microscope connected to a computer running $\mathrm{NIH}$ Image software (21). Nonoverlapping fields were sampled by a systematic serpentine progression across the whole fascicle. Cross-sectional profiles of myelinated axons were 
selected for calculation of axonal diameter and sorted into incremental size bins of $1 \mu \mathrm{m}$ before being grouped as small ( $4 \mu \mathrm{m}$ in diameter), medium ( $>4$ and $<8 \mu \mathrm{m}$ in diameter), and large ( $8 \mu \mathrm{m}$ in diameter) fibers. Between 1,000 and 3,000 fibers were counted per nerve segment. Fascicular perimeter and area were measured, and all blood vessels located in the epineurium and endoneurium were counted $(22,23)$.

Nerve conduction velocity measurements. Rats were anesthetized with either halothane (study 1) or isoflurane (study 2). Motor-nerve conduction velocity (MNCV) was measured from latencies of the compound $M$ waves of the electromyogram in the hind limb, and sensorynerve conduction velocity (SNCV) was measured from $\mathrm{H}$ reflex latencies in the same system. Preliminary studies involving selective transection of the dorsal but not ventral roots established the validity of our $\mathrm{H}$ wave measurements (our unpublished data), and $\mathrm{H}$ waves were routinely verified by their continued presence at stimulation voltages too low to elicit either $\mathrm{M}$ or $\mathrm{F}$ waves (23). Stimulation of the nerve was performed at the sciatic notch and Achilles tendon, and evoked electromyograms were recorded from the interosseus muscles of the foot. Mid-thigh nerve temperature was maintained at $36^{\circ} \mathrm{C} \pm 0.5^{\circ} \mathrm{C}$ throughout the procedure.

In situ bybridization and RT-PCR studies on expression of $\mathrm{Hb}$ proteins. In situ hybridization was performed on paraffin sections using a 642-bp ${ }^{33} \mathrm{P}-\mathrm{UTP}$-labeled mouse probe directed against $d h b$ (24). The probe was generated using T3 and T7 polymerases (Boehringer Mannheim), purified using a Microspin S-200 HR column (Amersham Biosciences, Arlington Heights, Illinois, USA), and precipitated overnight. Probes were hybridized overnight at $52^{\circ} \mathrm{C}$. After high-stringency washing, RNase treatment, and subsequent dehydration, sections were dipped in Kodak NTB-2 emulsion (Eastman Kodak Company, Rochester, New York, USA), exposed for approximately 21 days at $4^{\circ} \mathrm{C}$, and developed using Kodak D19 developer. To perform grain counts, five nerves were analyzed for both the diabetic and nondiabetic conditions. One transverse section from each nerve was hybridized for $d h b$ antisense. All hybridizations and emulsion incubations for the 10 nerve sections were performed at the same time and for the same amount of time in the same experiment. Sections were stained for hematoxylin and eosin after emulsion incubation. Three fields from each section were then scanned at $\times 400$ in both bright field (for tissue area) and dark field (for grain area) and digitized using Optimas 6.1 imaging software (Optimas Corp., Silver Spring, Maryland, USA). For each field, the areas occupied by tissue and by silver grains were recorded. Background for each nerve was determined by measuring the area occupied by silver grains in an equivalentsized field immediately adjacent to the section in question. The grain area/tissue area ratio was computed and averaged for the three fields to determine a value for each nerve, and background was subtracted. These individual nerve values were then averaged for each condition.

For quantitative RT-PCR, total RNA was isolated from $1-\mathrm{cm}$ mid-thigh segments of the sciatic nerve using Qiagen RNeasy (Chatsworth, California, USA). The RNA was subjected to reverse transcription, and the resulting cDNA was amplified by PCR using gene-specific primers and fluorescent probes (TaqMan; Applied Biosystems, Forest City, California, USA) for the test gene $d h b$ and the ubiquitously expressed gene gapdh. The test gene-specific probe was labeled with a different fluorophor (VIC; Applied Biosystems) from that used for gapdh (FAM; Applied Biosystems), allowing for quantification of both signals in the same reaction tube in a real-time PCR machine (ABI 7700; Applied Biosystems). The $d h b$ signal was normalized to the gapdh signal.

Statistical analyses. All data are presented as group means $\pm 1 \mathrm{SD}$. Differences between groups were analyzed by either unpaired $t$ test or ANOVA with the Student-Newman-Keuls post hoc test to identify specific between-group differences.

\section{Results}

General physiologic effects. SHh-IgG treatment was without effect on body weight or plasma glucose levels of control rats as compared with vehicle-treated controls. All animals were inspected daily, and no animals were withdrawn from the study because of either altered

Table 1

Physiologic and behavioral parameters of rats in study 1

\begin{tabular}{|c|c|c|c|c|c|c|c|}
\hline & $\begin{array}{l}\text { Final } \\
\text { plasma } \\
\text { glucose } \\
(\mathrm{mmol} / \mathrm{l})\end{array}$ & $\begin{array}{c}\text { Final } \\
\text { body } \\
\text { weight } \\
(\mathrm{g})\end{array}$ & $\begin{array}{l}\text { Thermal } \\
\text { response } \\
\text { latency } \\
\text { (s) }\end{array}$ & $\begin{array}{l}\text { Formalin- } \\
\text { evoked } \\
\text { flinches } \\
\text { (sum/h) }\end{array}$ & $\begin{array}{l}\text { Femoral- } \\
\text { artery } \\
\text { MSAP } \\
(\mathrm{mm} \mathrm{Hg})\end{array}$ & $\begin{array}{l}\text { Nerve } \\
\text { laser } \\
\text { Doppler } \\
\text { flux (AU) }\end{array}$ & $\begin{array}{c}\text { Nerve } \\
\text { vascular } \\
\text { conductance } \\
(\mathrm{AU} / \mathrm{mm} \mathrm{Hg})\end{array}$ \\
\hline Control plus vehicle & $9.0 \pm 2.5$ & $563 \pm 45$ & $14.1 \pm 2.5^{\mathrm{A}}$ & $10 \pm 8^{A}$ & $126 \pm 12$ & $46 \pm 7^{A}$ & $0.370 \pm 0.075^{\mathrm{A}}$ \\
\hline $\begin{array}{l}\text { Control plus SHh-lgG } \\
(1.0 \mathrm{mg} / \mathrm{kg})\end{array}$ & $8.8 \pm 2.5$ & $571 \pm 32$ & $14.0 \pm 3.4^{\mathrm{A}}$ & $7 \pm 6^{A}$ & $114 \pm 11$ & $44 \pm 7^{A}$ & $0.406 \pm 0.083^{\mathrm{A}}$ \\
\hline Diabetic plus vehicle & $40.1 \pm 6.2$ & $293 \pm 55$ & $18.1 \pm 2.4^{\mathrm{B}}$ & $72 \pm 15^{\mathrm{B}}$ & $97 \pm 27$ & $25 \pm 13^{B}$ & $0.257 \pm 0.103^{B}$ \\
\hline $\begin{array}{l}\text { Diabetic plus SHh-IgG } \\
(1.0 \mathrm{mg} / \mathrm{kg})\end{array}$ & $35.0 \pm 5.0$ & $282 \pm 40$ & $16.2 \pm 3.9$ & $77 \pm 16^{\mathrm{B}}$ & $101 \pm 18$ & $23 \pm 9^{B}$ & $0.230 \pm 0.073^{B}$ \\
\hline Statistical significance & & & $\begin{array}{l}P<0.05 \\
(A \text { vs. B) }\end{array}$ & $\begin{array}{l}P<0.01 \\
(A \text { vs. B) }\end{array}$ & & $\begin{array}{l}P<0.01 \\
(\text { A vs. } B)\end{array}$ & $\begin{array}{l}P<0.01 \\
(A \text { vs. } B)\end{array}$ \\
\hline
\end{tabular}

Data are given as means \pm 1 SD; $n=10-12$ per group. Statistical comparisons were made by ANOVA with the Student-Newman-Keuls post hoc test. Massive differences in body weight and glucose were not tested. MSAP, mean systemic arterial pressure; AU, arbitrary units. 
Table 2

Final plasma glucose and body weight data, motor (MNCV) and sensory (SNCV) conduction velocities, and sciatic-nerve NGF and NPY content for study 2

\begin{tabular}{|c|c|c|c|c|c|c|}
\hline & $\begin{array}{c}\text { Final plasma } \\
\text { glucose }(\mathrm{mmol} / \mathrm{l})\end{array}$ & $\begin{array}{l}\text { Final body } \\
\text { weight }(\mathrm{g})\end{array}$ & $\begin{array}{l}\text { Sciatic-nerve } \\
\operatorname{MNCV}(\mathrm{m} / \mathrm{s})\end{array}$ & $\begin{array}{l}\text { Sciatic-nerve } \\
\operatorname{SNCV}(\mathrm{m} / \mathrm{s})\end{array}$ & $\begin{array}{l}\text { Sciatic-nerve } \\
\operatorname{NGF}(\mathrm{pg} / \mathrm{cm})\end{array}$ & $\begin{array}{l}\text { Sciatic-nerve } \\
\text { NPY }(\mathrm{fmol} / \mathrm{cm})\end{array}$ \\
\hline Control plus vehicle & $9.9 \pm 0.4$ & $507 \pm 46$ & $60.4 \pm 2.4^{\mathrm{A}}$ & $58.7 \pm 3.7^{\mathrm{A}}$ & $50.9 \pm 22.8^{\mathrm{A}}$ & $118.0 \pm 19.7^{\mathrm{A}}$ \\
\hline Diabetic plus vehicle & $38.6 \pm 3.8$ & $298 \pm 31$ & $44.6 \pm 3.9^{\mathrm{B}}$ & $42.1 \pm 3.5^{B}$ & $26.6 \pm 13.0^{B}$ & $87.4 \pm 15.5^{\mathrm{B}}$ \\
\hline $\begin{array}{l}\text { Diabetic plus SHh-lgG } \\
(0.3 \mathrm{mg} / \mathrm{kg})\end{array}$ & $36.6 \pm 4.4$ & $298 \pm 43$ & $54.9 \pm 8.6^{C}$ & $55.1 \pm 4.2^{C}$ & $49.7 \pm 21.8^{c}$ & $109.6 \pm 10.9^{A}$ \\
\hline $\begin{array}{l}\text { Diabetic plus SHh-lgG } \\
(3.0 \mathrm{mg} / \mathrm{kg})\end{array}$ & $37.4 \pm 3.0$ & $326 \pm 45$ & $62.3 \pm 6.7^{\mathrm{D}}$ & $61.5 \pm 4.4^{\mathrm{D}}$ & $31.3 \pm 11.9^{\mathrm{D}}$ & $112.1 \pm 17.3^{\mathrm{A}}$ \\
\hline \multirow[t]{2}{*}{ Statistical significance } & & & $\begin{array}{c}P<0.01 \\
(\mathrm{~A}, \mathrm{C}, \text { and } \mathrm{D} \text { vs. }\end{array}$ & $\begin{array}{c}P<0.01 \\
A, \text { and } D \text { vs. } B)\end{array}$ & $\begin{array}{c}P<0.05 \\
(A \text { and } C \text { vs. } B)\end{array}$ & $\begin{array}{l}P<0.01 \\
(\text { A vs. B) }\end{array}$ \\
\hline & & & $\begin{array}{l}P<0.05 \\
\text { (C vs. D) }\end{array}$ & $\begin{array}{l}P<0.01 \\
\text { (C vs. D) }\end{array}$ & $\begin{array}{l}P<0.01 \\
\text { (C vs. D) }\end{array}$ & \\
\hline
\end{tabular}

Data are given as means $\pm 1 \mathrm{SD} ; n=8-10$ per group. Statistical comparisons were made by ANOVA with the Student-Newman-Keuls post hoc test. Massive differences in body weight and glucose were not tested.

general behavior or gross physical disorders. In all three studies, diabetic rats lost weight and were hyperglycemic. Treatment with SHh-IgG was without effect on the severity of diabetes as judged by these two indices (Tables 1, 2, and 3).

Hh expression in rat peripheral nerve. Peripheral nerve of diabetic rats showed a consistent reduction in mRNA for $d h b$ relative to nondiabetic controls. Using in situ hybridization with a dhh antisense probe, we saw localization of silver grains above Schwann cell nuclei in the endoneurium of the sciatic nerve of nondiabetic rats (Figure 1, $\mathrm{c}$ and e). The epineurium and perineurium did not show any clusters of sliver grains. A similar distribution was noted in nerve from diabetic rats, but with reduced signal intensity (Figure 1a). The reduction in signal intensity did not appear to be a consequence of a generalized reduction in gene expression in the peripheral nerve of diabetic rats, since the distribution and intensity of binding of an actin antisense probe was not obviously different between nondiabetic (Figure 1d) and diabetic (Figure 1b) rats. Hybridizations using $d h b$ sense as a control for nonspecific binding showed no signal when performed on adjacent sections, and there was no signal in any region of the sciatic nerve when a probe to $s h b$ was used (data not shown).

In order to quantitate $d h b$ expression, we performed grain counting on the sciatic-nerve sections used for in situ hybridizations and quantitative RT-PCR, standardized to gapdh for each sample of nerve. Both grain counting and RT-PCR confirmed a significant $(P<0.05$ by unpaired $t$ test) reduction of $d h b$ mRNA levels in the sciatic nerve of diabetic rats as compared with control rats (Figures 1e and 2).

Sensory responses. After 10 weeks of hyperglycemia, vehicle-treated rats displayed thermal hypoalgesia and hyperalgesia after injection of formalin into the paw (Table 1). SHh-IgG treatment was without effect on either thermal or formalin-evoked responses of control rats and did not affect hyperalgesia after formalin injection in diabetic rats. However, thermal hypoalgesia in diabetic rats was partially ameliorated by SHh-IgG treatment, so that response times were intermediate between those of control and those of diabetic rats and not significantly different from either group.

Table 3

Final plasma glucose and body weight data, NGF retrograde accumulation, and sciatic-nerve CGRP content for study 3

Control plus vehicle

Control plus SHh-lgG $(0.3 \mathrm{mg} / \mathrm{kg})$

Control plus SHh-lgG $(3.0 \mathrm{mg} / \mathrm{kg})$

Diabetic plus vehicle

Diabetic plus SHh-lgG $(0.3 \mathrm{mg} / \mathrm{kg})$

Diabetic plus SHh-lgG $(3.0 \mathrm{mg} / \mathrm{kg})$

Statistical significance

$\begin{array}{ccc}\begin{array}{c}\text { Final plasma } \\ \text { glucose }(\mathrm{mmol} / \mathrm{I})\end{array} & \begin{array}{c}\text { Final body } \\ \text { weight }(\mathrm{g})\end{array} & \begin{array}{c}\text { NGF accumulation } \\ (\mathrm{pg} / 18 \mathrm{~h})\end{array} \\ 8.7 \pm 0.5 & 534 \pm 70 & 485.5 \pm 216.8^{\mathrm{A}} \\ 8.9 \pm 0.4 & 518 \pm 58 & 619.9 \pm 98.3^{\mathrm{B}} \\ 8.9 \pm 0.4 & 534 \pm 55 & 392.9 \pm 136.3^{\mathrm{D}} \\ 38.9 \pm 3.8 & 301 \pm 54 & 198.2 \pm 105.1^{\mathrm{C}} \\ 37.4 \pm 4.2 & 289 \pm 45 & 365.0 \pm 275.1^{\mathrm{D}} \\ 39.6 \pm 4.5 & 301 \pm 52 & 313.4 \pm 187.9^{\mathrm{D}} \\ & & P<0.01 \\ & & (\text { A and B vs. C) } \\ & & P<0.05 \\ & & (\mathrm{~B} \text { vs. D) }\end{array}$

Sciatic-nerve
CGRP $(\mathrm{ng} / \mathrm{cm})$
$6.88 \pm 0.69^{\mathrm{A}}$
$7.85 \pm 0.87^{\mathrm{B}}$
$6.94 \pm 0.51^{\mathrm{C}}$
$4.03 \pm 0.58^{\mathrm{D}}$
$6.46 \pm 0.64^{\mathrm{E}}$
$6.00 \pm 0.74^{\mathrm{F}}$
$P<0.01$
$(\mathrm{~A}-\mathrm{C}, \mathrm{E}$, and Fvs. D)
$P<0.05$
$(\mathrm{~A}$ and D-F vs. B)
$P<0.05$
$(\mathrm{~A}$ and $\mathrm{C}$ vs. F)

Data are given as means $\pm 1 \mathrm{SD} ; n=8-10$ per group. Statistical comparisons were made by ANOVA with the Student-Newman-Keuls post hoc test. Massive differences in body weight and glucose were not tested. 


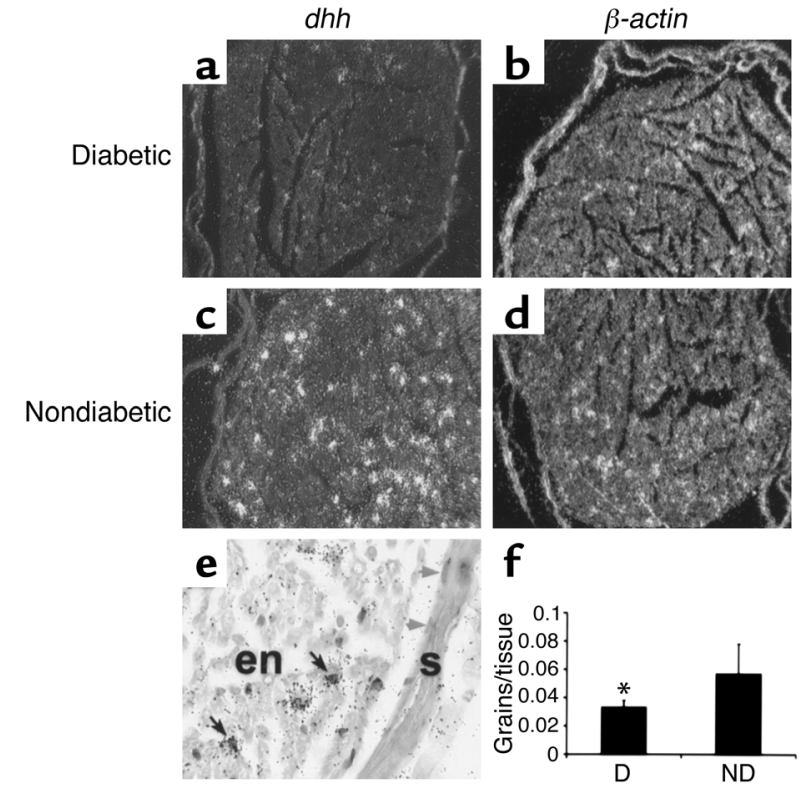

Figure 1

Reduced dhh mRNA in sciatic nerves of diabetic rats as shown by radioactive in situ hybridization. Hybridizations were performed on transverse sections of paraffin-embedded sciatic nerves from diabetic ( $\mathbf{a}$ and $\mathbf{b}$ ) and nondiabetic control ( $\mathbf{c}-\mathbf{e})$ rats using antisense probes for $d h h$ (a, c, and $\mathbf{e}$ ) or $\beta$-actin (b and $\mathbf{d})$. In (e), black arrows indicate signal above the nuclei of endoneurial Schwann cells, and gray arrows indicate nuclei of cells in the perineurial sheath that are unlabeled by the $d$ hh probe. Magnification, $\times 200$ (a-d); $\times 400$ (e). $\ln (\mathbf{f})$, quantification of $d h h$ grain area relative to tissue-section area is shown in diabetic and nondiabetic control nerves. Data are given as means $\pm \mathrm{SEM} ; n=5$ nerves per group. ${ }^{*} P<0.05$. D, diabetic; ND, nondiabetic; en, endoneurial; $s$, perineurial sheath.

Nerve conduction velocities. Diabetes impeded the progressive increase of both MNCV (Figure 3a) and SNCV (Figure $3 \mathrm{~b}$ ) seen in control animals over the 10-week study period, so that at the end of each experiment, $\mathrm{MNCV}$ and SNCV were significantly lower $(P<0.01)$ in vehicle-treated diabetic rats than in vehicle-treated controls (Figure 3 and Table 2). SHh-IgG treatment begun after 5 weeks of untreated diabetes and maintained for the subsequent 5 weeks reversed these deficits in a dose-dependent manner, with a partial effect noted with $0.3 \mathrm{mg} / \mathrm{kg}$ (Table 2) and complete efficacy with 1.0 (Figure 3 ) or $3.0 \mathrm{mg} / \mathrm{kg}$ (Table 3). SHh-IgG had no effect on NCV in control rats.

Sciatic-nerve laser Doppler flux and systemic arterial pressure. Diabetic rats were mildly hypotensive and had a 50\% reduction in sciatic-nerve laser Doppler flux (Table 1). To compensate for potentially decreased perfusion pressure and to assess vascular factors local to the sciatic nerve, vascular conductance was calculated and also showed a marked reduction in vehicle-treated diabetic rats as compared with vehicle-treated controls. Neither the central nor the local vascular changes in diabetic rats were affected by treatment with SHh-IgG at $1 \mathrm{mg} / \mathrm{kg}$, and this dose also did not alter vascular parameters in control rats.

Nerve structure. In the mid-thigh sciatic nerve, diabetes caused a significant decrease in the proportion of large myelinated axons ( $8 \mu \mathrm{m}$ in diameter) and a corresponding increase in the proportion of medium-sized ( $>4$ and $<8$ $\mu \mathrm{m}$ ) axons (Table 4). Treating diabetic rats with $1 \mathrm{mg} / \mathrm{kg}$ of SHh-IgG for the last 5 weeks of hyperglycemia normalized the size-frequency distribution, whereas there was no effect on the fiber size-frequency distribution of control rats. Neither diabetes nor treatment with $1 \mathrm{mg} / \mathrm{kg}$ of SHhIgG had any effect on the size (fascicular perimeter and area) or vascularization of the sciatic nerve (Table 4).

NGF and neuropeptides. Levels of NGF in the unligated sciatic nerve were reduced by approximately $50 \%$ in vehicle-treated diabetic rats as compared with vehicle-treated controls (Table 2). Treatment with SHh-IgG prevented this reduction, with the most marked effect being noted at the lower dose $(0.3 \mathrm{mg} / \mathrm{kg})$. This could arise from either normalization of NGF levels in steady-state retrograde axonal transport in sciatic-nerve axons or an increase in expression of NGF in Schwann or perineurial cells of the sciatic nerve. Diabetes reduced NGF retrograde transport, and this was ameliorated by SHh-IgG treatment, with the lower dose again being most effective (Table 3 ). The neuropeptides NPY (Table 2) and CGRP (Table 3) were both reduced in the unligated sciatic nerve of vehicle-treated diabetic rats as compared with vehicle-treated controls. SHh-IgG treatment prevented depletion of both NPY and CGRP in diabetic nerve at both the 0.3 and $3.0 \mathrm{mg} / \mathrm{kg}$ doses. SHh-IgG treatment also had effects on the nerve of control rats, with $0.3 \mathrm{mg} / \mathrm{kg}$ of SHh-IgG significantly increasing CGRP levels as compared with vehicle-treated controls, in association with a trend toward elevated retrograde transport of NGF (Table 3).

\section{Discussion}

DHh protein had previously been localized to Schwann cells of peripheral nerve in adult mice (6), and we have now extended these studies by confirming the distribution of mRNA for $d h b$ in adult rat nerve using in situ hybridization techniques. We also found a quantitative decrease in the amount of $d h b$ mRNA in sciatic nerve of rats made diabetic in early adulthood and maintained for a 10 -week period. In conjunction

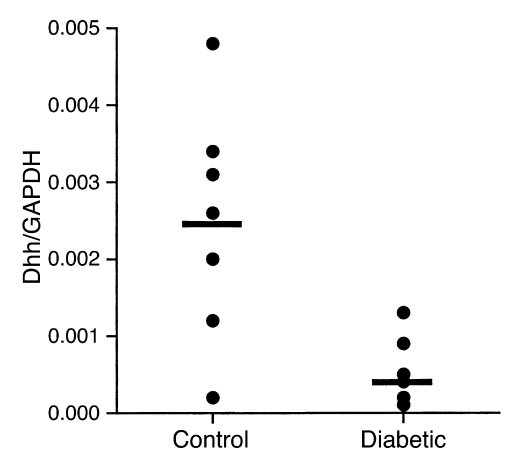

\section{Figure 2}

Reduced $d h h$ mRNA levels in sciatic nerves of diabetic rats as shown by quantitative RT-PCR ( $n=7$ per group). Each data point represents levels in a nerve segment from one animal, and horizontal bars represent group means, which were significantly different by unpaired $t$ test $(P<0.01)$. 

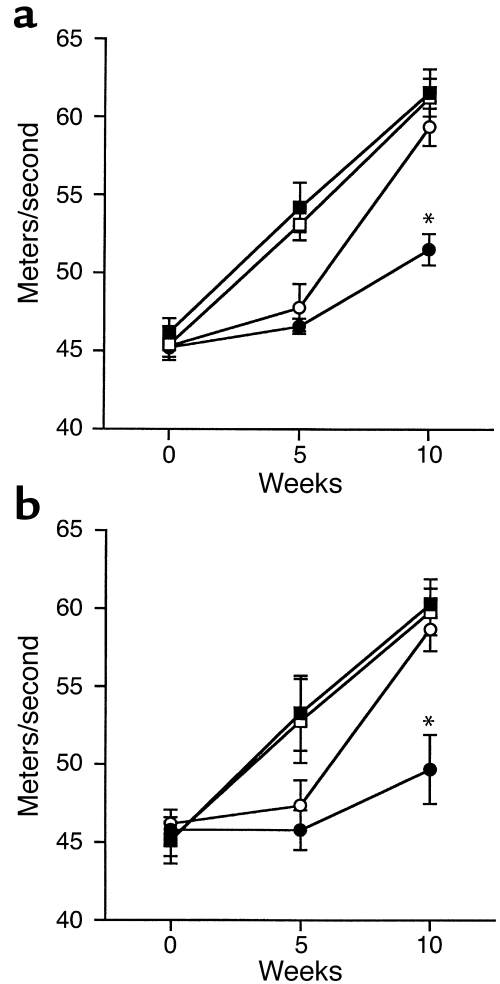

with reports of depletion of the Schwann cell-derived factor ciliary neuronotrophic factor (CNTF) and structural damage to Schwann cells in diabetic rats, cats, and humans (25-27), it appears that Schwann cell dysfunction is an early consequence of diabetes. Experimentally diabetic rodents rarely live long enough to develop the extreme degenerative and demyelinating pathologies seen in diabetic patients with neuropathy and more accurately model the early neurochemical disorders resulting from hyperglycemia. It is plausible that the metabolic disruption of Schwann cells that impairs $d h b$ expression may be an early pathogenic step in the progression of diabetic neuropathy. To address this issue and investigate whether $\mathrm{Hh}$ proteins have a role in the mature peripheral nervous system, we treated diabetic rats with exogenous $\mathrm{Hh}$ protein and determined efficacy against structural and functional disorders of the peripheral nervous system.

\section{Figure 3}

SHh-IgG treatment reverses conduction slowing in diabetic rats. $\mathrm{MNCV}(\mathbf{a})$ and SNCV (b) measurements were made in the same animals before the onset of diabetes, after 5 weeks of untreated diabetes, and after 5 weeks of thrice weekly treatment with $1 \mathrm{mg} / \mathrm{kg}$ of SHh-lgG or vehicle. Data are given as means \pm SEM; $n=10-12$ per group. Vehicle-treated controls are represented by filled squares, SHh-IgG-treated controls by open squares, vehicle-treated diabetics by filled circles, and SHh-lgG-treated diabetics by open circles. Statistical comparisons between groups were made at the 10-week time point by ANOVA followed by the Student-Newman-Keuls post hoc test. ${ }^{*} P<0.01$ for untreated diabetics versus all other groups.

For Hh replacement therapy, we used an SHh-IgG fusion protein that activates appropriate intracellular signaling pathways in vitro (our unpublished data) and enhances nerve regeneration in vivo (9). Any effects of this construct are unlikely to be caused by the IgG portion, as both SHh-IgG and polypropylene glycol-conjugated SHh have similar effects on nerve regeneration (9, 28). All members of the Hh family of secreted proteins bind to the transmembrane receptor patched (Ptc), thereby releasing inhibition by Ptc of the associated transmembrane signaling protein smoothened (Smo). The subsequent signal arising from disinhibition of Smo activates the Gli family of transcription factors (Gli-1, -2, and -3) that regulate expression of a growing, but incompletely characterized, number of genes. Both DHh and SHh exhibit similar binding affinities for Ptc, but SHh appears to have more marked biologic effects (29). Thus, while we originally identified a decrease in the mRNA for $d h b$ in the peripheral nerve of diabetic rats, it appeared appropriate to develop SHh as a potential therapy to replace DHh deficiency in order to obtain the greatest potential for in vivo activity.

Rats were made diabetic in early adulthood and exposed to 5 weeks of untreated hyperglycemia before initiation of treatment with SHh-IgG for a further 5 weeks. This paradigm allowed consideration of the effects of diabetes on the final stages of maturation of the peripheral nervous system, as illustrated by the progressive increase in the conduction velocity of control animals. Untreated diabetes halted this maturation process. The initiation of SHh-IgG treatment appeared to not only allow maturation to proceed but also to

Table 4

Nerve morphometric parameters of rats in study 1

\begin{tabular}{|c|c|c|c|c|c|c|c|}
\hline & \multicolumn{3}{|c|}{ Relative proportion of axons } & \multirow{2}{*}{$\begin{array}{c}\text { Endoneurial } \\
\text { vessels } \\
\text { per fascicle }\end{array}$} & \multirow{2}{*}{$\begin{array}{l}\text { Epineurial } \\
\text { vessels } \\
\text { per fascicle }\end{array}$} & \multirow{2}{*}{$\begin{array}{l}\text { Fascicular } \\
\text { perimeter } \\
\quad(\mathrm{mm})\end{array}$} & \multirow{2}{*}{$\begin{array}{c}\text { Fascicular } \\
\text { area } \\
\left(\mathrm{mm}^{2}\right)\end{array}$} \\
\hline & $\leq 4 \mu \mathrm{m}$ & $>4$ and $<8 \mu \mathrm{m}$ & $\geq 8 \mu \mathrm{m}$ & & & & \\
\hline Control plus vehicle & $19.4 \pm 6.2$ & $58.6 \pm 3.2^{\mathrm{A}}$ & $22.0 \pm 4.7^{\mathrm{A}}$ & $35 \pm 10$ & $40 \pm 16$ & $2.5 \pm 0.2$ & $0.51 \pm 0.06$ \\
\hline Control plus SHh-lgG $(1.0 \mathrm{mg} / \mathrm{kg})$ & $19.9 \pm 6.3$ & $56.9 \pm 3.6^{\mathrm{A}}$ & $23.2 \pm 4.5^{\mathrm{A}}$ & $41 \pm 8$ & $49 \pm 18$ & $2.6 \pm 0.2$ & $0.56 \pm 0.09$ \\
\hline Diabetic plus vehicle & $19.3 \pm 5.6$ & $64.9 \pm 4.6^{B}$ & $15.7 \pm 2.4^{\mathrm{B}}$ & $37 \pm 7$ & $48 \pm 17$ & $2.5 \pm 0.1$ & $0.48 \pm 0.05$ \\
\hline Diabetic plus SHh-lgG $(1.0 \mathrm{mg} / \mathrm{kg})$ & $18.2 \pm 3.8$ & $60.0 \pm 2.9^{\mathrm{A}}$ & $21.8 \pm 4.7^{\mathrm{A}}$ & $38 \pm 6$ & $50 \pm 23$ & $2.5 \pm 0.1$ & $0.51 \pm 0.04$ \\
\hline Statistical significance & & $\begin{array}{l}P<0.05 \\
(A \text { vs. B) }\end{array}$ & $\begin{array}{l}P<0.05 \\
(A \text { vs. B })\end{array}$ & & & & \\
\hline
\end{tabular}

Data are given as means $\pm 1 \mathrm{SD} ; n=6-11$ per group. Statistical comparisons were made by ANOVA with the Student-Newman-Keuls post hoc test. 
dose-dependently enhance it, so that the conduction velocity of treated diabetic rats was restored to control values over the 5-week treatment period. Both MNCV and SNCV disorders were corrected by SHh-IgG, indicating effects on the function of large myelinated sensory and motor fibers. As in the vehicle-treated diabetic animals, the SHh-IgG-treated diabetic animals lost body weight and remained hyperglycemic, showing that the effects of SHh treatment on nerve function in diabetic rats were not due to a global amelioration of the diabetic condition. Although large-fiber conduction slowing in diabetic rodents has been used extensively as an index of nerve dysfunction that equates the model to human diabetic neuropathy, the precise etiology of the disorder remains unclear. The successful protection of conduction velocities in diabetic rats by a legion of agents that prevent reduced blood flow in peripheral nerve has promoted the hypothesis that conduction deficits arise secondary to ischemic hypoxia (30). However, the dramatic effects of SHh-IgG on conduction velocity in diabetic rats were not accompanied by improved nerve blood flow or vascularization, suggesting an effect either downstream of, or unconnected to, nerve ischemia.

Diabetes-induced slowing of large-myelinated-fiber conduction was accompanied by a decrease in the proportion of the largest fibers present in the sciatic nerve. There is no marked fiber loss in short-term diabetic rats, and the reduction in the proportion of large fibers was accompanied by a parallel increase in the proportion of medium-sized fibers, suggesting either delayed radial growth of axons or atrophy of large axons. As axonal caliber is a major determinant of conduction velocity, the capacity of SHh-IgG to restore or maintain the proportion of large myelinated fibers in the sciatic nerve of diabetic rats reveals a potential mechanism that could explain the effect of SHh-IgG on conduction. Radial growth of axons and maintenance of axonal caliber is determined by neurofilament proteins and their phosphorylation status (31-34). Defects in neurofilament gene expression, synthesis, axonal transport, and phosphorylation have all been implicated in the decreased axonal caliber seen in diabetic rodents $(19,35-37)$. The etiologic cascade linking insulin deficiency and hyperglycemia with neurofilament disorders in diabetes is unresolved and could reflect direct effects on neuronal cell bodies (38). Alternatively, there is some evidence that neurofilament organization and axonal caliber are under the local control of Schwann cell-derived factors (39), which are also a target for the metabolic disruption induced by hyperglycemia. It will be of interest to determine whether Hh proteins have effects on neurofilament gene expression or posttranslational organization.

The efficacy of the SHh-IgG fusion protein also extended to the protection of small fibers in diabetic rats, as indicated by partial amelioration of thermal hypoalgesia and maintenance of nerve neuropeptide levels. It is well established that a deficit in supply, uptake, and retrograde axonal transport of NGF from the target tissues to small sensory fibers contributes to diminished synthesis and anterograde axonal transport of both substance $P$ and CGRP in the peripheral nerves of diabetic rats (40). NPY expression in sympathetic neurons is also under the influence of target-derived NGF (41), and diabetesinduced NGF depletion is accompanied by a reduction in sciatic-nerve NPY levels (42). It is possible that disruption of NGF supply to sympathetic nerves of the nervi nervorum results in this decrease in sciatic NPY levels. Normalization of NGF supply and delivery by SHh-IgG treatment could thus account for maintenance of both CGRP and NPY levels in sensory and sympathetic components of the sciatic nerve. However, the extent to which sympathetic ganglia are deprived of NGF in diabetic rats is unresolved $(43,44)$, and, unlike for sensory nerves, a causative association between NGF depletion and disorders of sympathetic nerves has yet to be established in diabetes.

The mechanisms by which systemically delivered SHhIgG fusion protein protects diabetic nerve remain to be resolved. The broad distribution of Ptc and Smo in nerve and other tissues suggests the potential for direct or indirect effects on peripheral neurons or a combination of the two. We have some evidence, using mice where a $\beta$-galactosidase transgene has been inserted under control of the Ptc-1 promoter, that systemic delivery of SHh-IgG can activate signaling in peripheral nerve (our unpublished data), suggesting direct access to cells within the nerve trunk. SHh induces mRNA for brain-derived neurotrophic factor (BDNF) and IGF-1 in responsive fibroblast cell lines and primary epi- and perineurial-derived fibroblasts in vitro (45), both of which prevent aspects of nerve dysfunction when delivered exogenously to hyperglycemic rats $(21,46)$. Hh-responsive epi- and perineurial cells and endoneurial fibroblasts could therefore provide a source of local trophic support for axons in SHh-IgG-treated diabetic nerves. Cells external to the nerve trunk could also provide neurotrophic support to peripheral nerves. For example, follicular keratinocytes produce NGF local to the free nerve endings of sensory neurons in the epidermis (47). These cells also express mRNA for the Hh receptor Ptc (48) and could be induced by SHh treatment to produce NGF that is then taken up by neuronal terminals and retrogradely transported to the perikaryon. Indeed, the increased NGF undergoing transport in control rats treated with SHh$\operatorname{IgG}$, and the accompanying increase in nerve CGRP levels, suggests that NGF production in the skin may be tightly regulated by $\mathrm{Hh}$ signaling pathways.

Our studies show persistent expression of $d h b$ in the postnatal endoneurium that may reflect an ongoing role for the Hh pathway in the maturation and maintenance of the adult peripheral nervous system. They also imply that impairment of DHh production by diabetes may contribute to the development of neurochemical, functional, and structural disorders of sensory, motor, and autonomic nerves indicative of diabetic neuropathy. The specific pathogenic mechanism linking hyperglycemia with reduced $d h b$ gene expression requires clarification and may reflect a general disruption of perineurial and Schwann cell-derived support functions. The efficacy of 
the SHh-IgG fusion protein in the absence of obvious physiologic consequences in control rats also highlights a therapeutic potential for diabetic neuropathy. The broad efficacy of SHh-IgG, as compared with other neurotrophic factors that target specific neuronal populations, may be of particular advantage when considering treatment of indiscriminate peripheral neuropathies such as diabetic neuropathy. Thus, although further studies are clearly required, SHh-IgG exhibits significant potential as a therapy for diabetic peripheral neuropathy.

\section{Acknowledgments}

This work was supported by Biogen Inc., Curis Inc., and National Institutes for Health grant NS38855 (to N.A. Calcutt). We thank John Lydon for histology and grain counting expertise and Allison Berg and Carmen Pepicelli for assistance with the in situ hybridization.

1. Bitgood, M.J., and McMahon, A.P. 1995. Hedgehog and BMP genes are coexpressed at many diverse sites of cell-cell interaction in the mouse embryo. Dev. Biol. 172:126-138.

2. Roelink, H., et al. 1994. Floor plate and motor neuron induction by Vhh-1, a vertebrate homologue of hedgehog expressed by the notochord. Cell. 76:761-775.

3. Wang, M.Z., et al. 1995. Induction of the dopaminergic neuron phenotype in the midbrain by sonic hedgehog protein. Nat. Med. 1:1184-1188.

4. Chiang, C., et al. 1996. Cyclopia and defective patterning in mice lacking sonic hedgehog gene function. Nature. 383:407-413.

5. Goodrich, L., and Scott, M. 1998. Hedgehog and patched in neural development and disease. Neuron. 21:1243-1257.

6. Parmantier, E., et al. 1999. Schwann cell-derived desert hedgehog controls the development of peripheral nerve sheaths. Neuron. 23:713-724.

7. Umehara, F., et al. 2000. A novel mutation of desert hedgehog in a patient with 46,XY partial gonadal dysgenesis accompanied by minifascicular neuropathy. Am. J. Hum. Genet. 67:1302-1305.

8. Berg, A., et al. 2000. Upregulation of desert hedgehog and hedgehog signaling during peripheral nerve regeneration. Soc. Neurosci. (Abstracts on CD-ROM). Abstr. 25; program no. 792.13.

9. Engber, T.M., et al. 2000. Sonic hedgehog improves functional recovery following sciatic nerve crush injury. Soc. Neurosci. (Abstracts on CDROM). Abstr. 25; program no. 792.14

10. Pola, R., et al. 2001. The morphogen sonic hedgehog is an indirect angiogenic agent upregulating two families of angiogenic growth factors. Nat. Med. 7:706-711.

11. Johnson, P.C., Brendel, K., and Meezan, E. 1981. Human diabetic perineurial cell basement membrane thickening. Lab. Invest. 44:265-270.

12. Ghani, M., et al. 1999. Perineurial abnormalities in the spontaneously diabetic dog. Acta Neuropathol. 97:98-102.

13. Pitre, D.A., Seifert, J.L., and Bauer, J.A. 2001. Perineurium inflammation and altered connexin isoform expression in a rat model of diabetes related peripheral neuropathy. Neurosci. Lett. 303:67-71.

14. Rechthand, E., Smith, Q.R., Latker, C.H., and Rapoport, S.I. 1987. Altered blood-nerve barrier permeability to small molecules in experimental diabetes mellitus. J. Neuropathol. Exp. Neurol. 46:302-314.

15. Taylor, F.R., et al. 2001. Enhanced potency of human sonic hedgehog by hydrophobic modification. Biochemistry. 40:4359-4371.

16. Calcutt, N.A., et al. 1999. Prosaposin gene expression and the efficacy of a prosaposin-derived peptide in preventing structural and functional disorders of peripheral nerve in diabetic rats. J. Neuropathol. Exp. Neurol. 58:628-636.

17. Calcutt, N.A., Li, L., Yaksh, T.L., and Malmberg, A.B. 1995. Different effects of two aldose reductase inhibitors on nociception and prostaglandin E. Eur. J. Pharmacol. 285:189-197.

18. Calcutt, N.A., Mizisin, A.P., and Kalichman, M.W. 1994. Aldose reductase inhibition, Doppler flux and conduction in diabetic rat nerve. Eur. J. Pharmacol. 251:27-33.

19. Fernyhough, P., et al. 1995 . Human recombinant nerve growth factor replaces deficient neurotrophic support in the diabetic rat. Eur. J. Newrosci. 7:1107-1110.

20. Malcangio, M., Garrett, N.E., and Tomlinson, D.R. 1995. Nerve growth factor treatment increases stimulus-evoked release of sensory neuropeptides in the rat spinal cord. Eur. J. Neurosci. 9:1101-1104.

21. Mizisin, A.P., et al. 1997. BDNF attenuates functional and structural disorders in nerves of galactose-fed rats. J. Neuropathol. Exp. Neurol. 56:1290-1301.
22. Weibel, E.R. 1979. Stereological methods. Academic Press. New York, New York, USA. 415 pp.

23. Mizisin, A.P., Steinhardt, R.C., O’Brien, J.S., and Calcutt, N.A. 2001. TX14(A), a prosaposin-derived peptide, reverses established nerve disorders in streptozotocin-diabetic rats and prevents them in galactosefed rats. J. Neuropathol. Exp. Neurol. 60:953-960.

24. Echelard, Y., et al. 1993. Sonic hedgehog, a member of a family of putative signaling molecules, is implicated in the regulation of CNS polarity. Cell. 75:1417-1430.

25. Calcutt, N.A., Muir, D., Powell, H.C., and Mizisin, A.P. 1992. Reduced ciliary neuronotrophic factor-like activity in nerves from diabetic or galactose-fed rats. Brain Res. 575:320-324.

26. Kalichman, M.W., Powell, H.C., and Mizisin, A.P. 1998. Reactive, degenerative, and proliferative Schwann cell responses in experimental galactose and human diabetic neuropathy. Acta Neuropathol. 95:47-56.

27. Mizisin, A.P., Shelton, G.D., Wagner, S., Rusbridge, C., and Powell, H.C. 1998. Myelin splitting, Schwann cell injury and demyelination in feline diabetic neuropathy. Acta Neuropathol. 95:171-174.

28. Pepinsky, R.B., et al. 2002. Long-acting forms of sonic hedgehog with improved pharmacokinetic and pharmacodynamic properties are efficacious in a nerve injury model. J. Pharmaceut. Sci. 91:371-387.

29. Pathi, S., et al. 2001. Comparative biological responses to human sonic, indian, and desert hedgehog. Mech. Dev. 106:107-117.

30. Cameron, N.E., Eaton, S.E., Cotter, M.A., and Tesfaye, S. 2001. Vascular factors and metabolic interactions in the pathogenesis of diabetic neuropathy. Diabetologia. 44:1973-1988.

31. Friede, R., and Samorajski, T. 1970. Axon caliber related to neurofilaments and microtubules in sciatic nerve. Anat. Rec. 167:379-387.

32. Hoffman, P.N., et al. 1987. Neurofilament gene expression: a major determinant of axonal caliber. Proc. Natl. Acad. Sci. USA. 84:3472-3476.

33. Nixon, R.A., Paskevich, P.A., Sihag, R.K., and Thayer, C.Y. 1994. Phosphorylation on carboxyl terminus domains of neurofilament proteins in retinal ganglion cell neurons in vivo: influences on regional neurofilament accumulation, interfilament spacing and axonal caliber. J. Cell Biol. 126:1031-1046.

34. Sanchez, I., et al. 2000. Local control of neurofilament accumulation during radial growth of myelinating axons in vivo: selective role of sitespecific phosphorylation. J. Cell Biol. 151:1013-1024.

35. Medori, R., Autilio-Gambetti, L., Monaco, S., and Gambetti, P. 1985. Experimental diabetic neuropathy: impairment of slow transport with changes in axon cross-sectional area. Proc. Natl. Acad. Sci. USA. 82:7716-7720.

36. Fernyhough, P., et al. 1999. Aberrant neurofilament phosphorylation in sensory neurons of rats with diabetic neuropathy. Diabetes. 48:881-889.

37. Yagihashi, S., Kamijo, M., and Watanabe, K. 1990. Reduced myelinated fiber size correlates with loss of axonal neurofilaments in peripheral nerve of chronically streptozotocin diabetic rats. Am. J. Pathol. 136:1365-1373.

38. Scott, J.N., Clark, A.W., and Zocodne, D.W. 1999. Neurofilament gene expression in progressive experimental diabetes: failure of synthesis and export by sensory neurons. Brain. 122:2109-2117.

39. de Waegh, A.M., Lee, V.M.-Y., and Brady, S.T. 1992. Local modulation of neurofilament phosphorylation, axonal caliber, and slow axonal transport by myelinating Schwann cells. Cell. 68:451-463.

40. Tomlinson, D.R., Fernyhough, P., and Diemel, L.T. 1997. Role of neurotrophins in diabetic neuropathy and treatment with nerve growth factors. Diabetes. 46(Suppl. 2):S43-S49.

41. Shadiack, A.M., Sun, Y., and Zigmond, R.E. 2001. Nerve growth factor antiserum induces axotomy-like changes in neuropeptide expression in intact sympathetic and sensory neurons. J. Neurosci. 21:363-371.

42. Hounsom, L., Corder, R., Patel, J., and Tomlinson, D.R. 2001. Oxidative stress participates in the breakdown of neuronal phenotype in experimental diabetic neuropathy. Diabetologia. 44:424-428.

43. Hellweg, R., and Hartung, H.D. 1990. Endogenous levels of nerve growth factor (NGF) are altered in experimental diabetes mellitus: a possible role for NGF in the pathogenesis of diabetic neuropathy. J. Neurosci. Res. 26:258-267.

44. Schmidt, R.E., et al. 2000. Effect of streptozotocin-induced diabetes on NGF, P75(NTR) and TrkA content of prevertebral and paravertebral rat sympathetic ganglia. Brain Res. 867:149-156.

45. Allendoerfer, K.L., Taratuska, A., and Drake, E.J. 2001. Sciatic nerve periand endoneurial fibroblasts respond to hedgehog in vitro. Soc. Neurosci. (Abstracts on CD-ROM). Abstr. 26; program no. 351.9.

46. Ishii, D.N. 1995. Implication of insulin-like growth factors in the pathogenesis of diabetic neuropathy. Brain Res. Brain Res. Rev. 20:47-67.

47. Raychaudhuri, S.P., Jiang, W.Y., and Farber, E.M. 1998. Psoriatic keratinocytes express high levels of nerve growth factor. Acta Derm. Venereol. 78:84-86.

48. Oro, A.E., et al. 1997. Basal cell carcinomas in mice overexpressing sonic hedgehog. Science. 276:817-821. 